\title{
Electric field effects on ionospheric and thermospheric parameters above the EISCAT station for summer conditions
}

\author{
V. V. Klimenko ${ }^{1}$, Yu. N. Korenkov ${ }^{1}$, M. Förster ${ }^{2}$ \\ ${ }^{1}$ West Department of IZMIRAN, Kaliningrad Observatory, Academy of Sciences, Russia ${ }^{2}$ GeoForschungsZentrum, Projektbereich 2.3, \\ Telegrafenberg D-14473 Potsdam, Germany Fax : + 49331288 1238; e-mail: mf@gfz- Potsdam.de
}

Received: 2 October 1997 / Revised: 6 April 1998/Accepted: 8 April 1998

\begin{abstract}
Numerical calculations of the thermospheric and ionospheric parameters above EISCAT are presented for quiet geomagnetic conditions in summer. The Global Self-consistent Model of the Thermosphere, Ionosphere and Protonosphere (GSM TIP) was used. The numerical results were obtained both with a selfconsistent calculation of the electric fields of magnetospheric and dynamo-action origin and with the magnetospheric electric fields only. It was found that the dynamo-electric field has some effect on the ionospheric convection pattern during quiet geomagnetic conditions. It has a marked effect mainly on the zonal neutral wind component above EISCAT $( \pm 20 \mathrm{~m} / \mathrm{s}$ at $140 \mathrm{~km}$ altitude). We have studied the effects of various fieldaligned current (FAC) distributions on thermosphere/ ionosphere parameters and we show that a qualitative agreement can be obtained with region-I and -II FAC zones at $75^{\circ}$ and $65^{\circ}$ geomagnetic latitude, respectively. The maximum FAC intensities have been assumed at 03-21 MLT for both regions with peak values of $2.5 \times 10^{-7} \mathrm{~A} \mathrm{~m}^{-2}$ (region I) and $1.25 \times 10^{-7} \mathrm{~A} \mathrm{~m}^{-2}$ (region II). These results are in agreement with statistical potential distribution and FAC models constructed by use of EISCAT data. The lack of decreased electron density in the night-time sector as observed by the EISCAT radar was found to be due to the spatial distribution of ionospheric convection resulting from electric fields of magnetospheric origin.
\end{abstract}

Key words. Electric fields and currents - Ionosphereatmosphere interactions. Modelling and forecasting

\section{Introduction}

It is well known that the coupling of the ionosphere and magnetosphere plays an important role in the large-scale

Correspondence to: M. Förster distributions of the ionosphere-magnetosphere-thermosphere parameters. The electric fields of magnetospheric and dynamo origin cause modifications of both the neutral atmosphere (temperature, density and composition) and the ionospheric parameters (electron and ion densities, electron and ion temperatures). These modifications influence each other through the ion-neutral dynamics and this topic remains of considerable interest.

The EISCAT Common Program 1 (CP-1) is capable of providing the three-dimensional ion velocity and other ionospheric parameters with good time resolutions of their diurnal variations. The direct measurements of the plasma velocity vectors give access to spatial distributions of the convection electric fields. Techniques have been developed to derive potential patterns from measurements of plasma velocity as a function of space and time. Ionospheric potential models deduced from EISCAT data were presented, e.g., by Fontaine et al. (1986), Foster (1987) and Senior et al. (1990). Recently, the statistical spatial distribution of the horizontal and field-aligned currents inferred from EISCAT data was presented by Fontaine and Peymirat (1996). These investigations show that for very quiet geomagnetic conditions the electric fields above the EISCAT station are small almost throughout the whole day with the exception of the evening to night-time hours from 18 to 24 LT. Therefore we can suppose that the dynamo electric fields could have a marked effect on the thermosphere-ionosphere parameters above EISCAT under geomagnetically quiet conditions.

The variability of the field-aligned currents (FAC), spatial distribution of the FAC zones and therefore of the ionospheric convection pattern can cause changes in the spatial distribution of the electron concentration in the high-latitude ionosphere in the evening/night sector above EISCAT. In this case the electric field of magnetospheric origin above EISCAT may be small, only a few $\mathrm{mV} / \mathrm{m}$, but the diurnal variation of the electron concentration is affected by the spatial distribution of the electron concentration over the whole polar region. Numerical models of the ionosphere have 
been used in a number of studies for the interpretation of the ionospheric processes above EISCAT (Farmer et al., 1990; Blelly et al., 1996a,b; Schoendorf et al., 1996; Namgaladze et al., 1996; Korenkov et al., 1996). These studies have shown the capability of numerical models to simulate the polar ionospheric structure above EISCAT, but some adjustments of the external inputs are needed to achieve good agreement with experimental EISCAT data.

It is well known that ionospheric convection is of special importance for the polar ionospheric dynamics, and its determination as model input parameter plays a significant role in ionospheric high-latitude modelling. The choice of the correct convection pattern and the subsequent numerical calculation of spatial electron density distributions for the polar region is a quite difficult problem. Therefore, some model calculations of the ionospheric structure above EISCAT were obtained under the assumption that ionospheric convection fields could be neglected at all under geomagnetically quiet conditions (see Blelly et al., 1996a,b). In the studies of Schoendorf et al. (1996) and Farmer et al. (1990) empirical models of the electric field pattern in the polar region were used. In contradistinction to these studies, we make use of FAC distributions as model input parameter for the self-consistent calculation of the electric field distribution in the high-latitude region.

In the paper of Korenkov et al. (1996) the influence of convection electric field patterns resulting from different FAC distributions on the ionospheric parameters above EISCAT was studied, but without special interest to the dynamo electric field effects. For only two time-sectors, we compared there model results with experimental EISCAT data except for the drift measurements.

The present work makes an effort to study the effects of the dynamo electric field on the thermosphereionosphere parameters above EISCAT using the numerical global model GSM TIP (Namgaladze et al. $1998,1990,1991)$. The second goal of the paper is to study the effect of magnetospheric electric fields on the spatial and temporal parameter distribution in order to determine the origin of the $N_{e}$ enhancements above EISCAT in the night-time sector and to obtain a best fitting of the FAC distributions necessary for a good agreement with the diurnal variation of $\mathrm{NmF} 2$ above EISCAT for geomagnetically quiet days. In doing so, we attempted to achieve an agreement with statistical models of FAC distributions and convection electric field patterns which were obtained from experimental EISCAT data as well. Calculations were performed for quiet geomagnetic conditions during summer solstice and high solar activity.

\section{Observational basis}

For this study we used EISCAT CP-1 registrations from intervals near summer solstices of the years 1989-1992.

CP-1 data are obtained with a fixed transmitting antenna in Tromsø (Norway), pointing approximately along the geomagnetic field direction. The three-dimensional velocity and anisotropy in other parameters are measured by means of the receiving stations at Kiruna (Sweden) and Sodankylä (Finland) with a common scattering volume at F-region heights $(278.5 \mathrm{~km}$ altitude). CP-1 is capable of providing very good time resolution down to the basic time-intervals of $5 \mathrm{~s}$. Here we use the EISCAT standard routine with integration times of 5 min for each data point.

The time-period under study is generally characterized by high to moderate solar activity $\left(F_{10.7}=135-277\right)$. The CP-1 intervals selected - as listed in Table 1 - are periods near the summer solstices where the geomagnetic activity was generally very low.

Figure 1 shows an overview about these observations as 'clouds' of data points together with 1-h-binned median values and their r.m.s. deviations indicated by

Table 1. CP-1 intervals selected for Fig. 1 together with some activity indices

\begin{tabular}{llllr}
\hline ddmmyy & $F_{10.7}$ & $\overline{F_{10.7}}$ & $\sum K p$ & Ap \\
\hline 010889 & 198.5 & 220 & $12-$ & 6 \\
020889 & 201.7 & 220 & $12-$ & 6 \\
050690 & 153.7 & 190 & $11 \circ$ & 5 \\
020790 & 276.6 & 185 & $13+$ & 6 \\
030790 & 253.8 & 185 & $14-$ & 7 \\
100791 & 200.2 & 215 & $25-$ & 19 \\
030892 & 135.1 & 130 & $8+$ & 4 \\
\hline
\end{tabular}

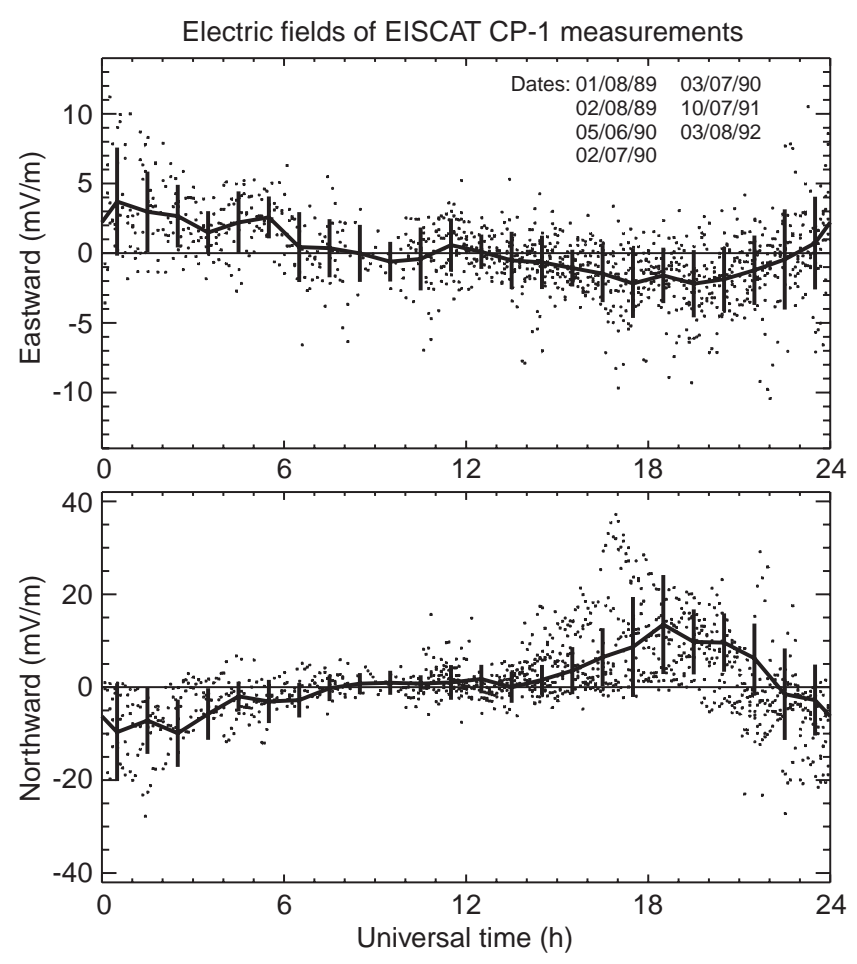

Fig. 1. Averaged EISCAT observations of the eastward (upper panel) and northward (bottom) electric field components for summer periods during geomagnetically quiet and high to medium solar activity conditions 
the thick line with the vertical bars. The upper panel shows the eastward electric field component (corresponding to northward drift with $1 \mathrm{mV} / \mathrm{m} \approx 20 \mathrm{~m} / \mathrm{s}$ ) and the lower panel the northward component (westward drift).

The experimental drift velocities can easily be divided into two different characteristic intervals: the daytime period between about 03 and 16 UT and the evening/ night-time period between about 16 and 03 UT. The electric fields during the first-time span are small; they do not exceed $5 \mathrm{mV} / \mathrm{m}$ (about $100 \mathrm{~m} / \mathrm{s}$ ). In the second time-span, the eastward electric field component (upper panel in Fig. 1) is also small, but the northward component (lower panel) may be as great as $15 \mathrm{mV} / \mathrm{m}$ (about $300 \mathrm{~m} / \mathrm{s}$ ) as the peak mean values in the eveningnight-time sectors show. The change of zonal drift velocity direction takes place in the evening sector near $23 \mathrm{LT}$. Thus we can suppose that the EISCAT station is located during daytime in a latitudinal range where the dynamo electric field can have comparable values with respect to the magnetospheric convection electric field.

\section{Brief description of the model GSM TIP}

The Global Selfconsistent Model of the Thermosphere, Ionosphere, and Protonosphere (GSM TIP) was described in detail by Namgaladze et al. (1988, 1990, 1991). It comprises several computational blocks for the description of the thermosphere, ionosphere, plasmasphere, and near-Earth magnetosphere in a height range from $80 \mathrm{~km}$ to 15 Earth radii including electromagnetic coupling processes as a single system. The model takes into account the offset between the geomagnetic and the geographic poles.

For a given set of input data (possibly time-dependent) the GSM TIP model calculates the time-dependent global three-dimensional structure of the neutral gas temperature $T_{n}$, mass density, wind velocity vector $V_{n}$ and number density of the main neutral gas components $\mathrm{N}_{2}, \mathrm{O}_{2}$ and $\mathrm{O}$ for the height range from 80 to $520 \mathrm{~km}$ by means of numerical integration of the corresponding three-dimensional continuity, momentum and heat balance equations in a spherical geomagnetic coordinate system.

In the ionospheric-protonospheric modules, parameters of the E, F1 and F2 region of the ionosphere and of the plasmasphere are calculated, namely, molecular ions $n\left(X^{+}\right)=n\left(N O^{+}\right)+n\left(\mathrm{O}_{2}^{+}\right)+n\left(N_{2}^{+}\right)$, atomic oxygen $n\left(O^{+}\right)$and hydrogen ion number densities $n\left(H^{+}\right)$, as well as ion $\left(T_{i}\right)$ and electron temperatures $\left(T_{e}\right)$, and ion velocities. The plasma drift caused by crossed electric and magnetic fields is taken into account.

The equation for the potential of the electric field $E=-\operatorname{grad}(\phi)$ is solved in the fourth programme module including the dynamo action of the thermospheric winds and magnetospheric sources via the aurora FACs. The spherical geomagnetic system is used in this block.

The spatial steps of the numerical integration are $10^{\circ}$ in geomagnetic latitude for the thermosphere and lower ionosphere modules, $5^{\circ}$ for the ionospheric-protono- spheric and electrical potential modules, and $15^{\circ}$ in the geomagnetic longitude for all groups of the model equations.

\section{Model Inputs}

In order to simulate the thermosphere-ionosphere parameters the GSM TIP requires the following inputs: solar UV and EUV spectra, FACs connecting the ionosphere with the magnetosphere and ionizing particle precipitation patterns and intensities.

For the solar EUV fluxes we have used the technique of Nusinov (1984) to construct the flux spectra for the period under study. Solar UV fluxes are as the Mount and Rottman (1983) data.

The ionospheric polar cap convection pattern is controlled by the specific FAC distributions. Region-I FACs are located near the boundary of the polar cap and region-II near the equatorial boundary of the auroral oval (Iijima and Potemra, 1976). In our model, the polar cap boundary was chosen at a constant geomagnetic latitude of $75^{\circ}$ while the location of regionII FACs was different for the various model runs. The FAC parameters used for the numerical calculations as input parameters are presented in Table 2. The argumentation for our choice of these specific FAC inputs will be given in the next sections.

The electron precipitation patterns in the auroral zone $\left(65^{\circ}-75^{\circ}\right.$ geomagnetic latitude) have been assumed in the following simple form according to precipitation measurements typical for quiet geomagnetic activity conditions (cf. Hardy et al., 1985). The soft electron precipitation belts with a characteristic energy of $0.2 \mathrm{keV}$ and an energy flux of $\sim 0.2 \mathrm{erg} \mathrm{cm}^{-2} \mathrm{~s}^{-1}$ have a Gaussian shape in the latitudinal direction and were centred around $+70^{\circ}$ and $-70^{\circ}$ geomagnetic latitude and equally distributed in the longitudinal direction. Energetic electrons with $E_{o}=3 \mathrm{keV}$ and maximal fluxes of $\sim 7 \mathrm{erg} \mathrm{cm}^{-2} \mathrm{~s}^{-1}$ at 02 MLT have Gaussian distribution forms in latitudinal and longitudinal directions. The spectral characteristic of the soft and the energetic precipitating electrons is chosen according to Maxwellian energy distributions.

Constant values of the concentrations of $\mathrm{N}_{2}, \mathrm{O}_{2}, \mathrm{O}$ and $T_{n}$ are imposed at the lower boundary $(80 \mathrm{~km})$ for the thermosphere equations, i.e. without tidal modes from the lower thermosphere.

All other model input data have been chosen as in the paper of Korenkov et al. (1996). It should be noted here that in this study we did not take into account the effect of the vibrationally excited nitrogen on the loss rate for the $\mathrm{O}^{+}$ions.

\section{Results of numerical modelling}

In this section we confine ourselves to model runs of one special day of the period under study. We selected 2-3 July 1990 to investigate the dynamo electric field and ionospheric convection effect on neutral wind velocities 
Table 2. Parameters of the field-aligned currents used as input for the different numerical model runs and their calculated potential drops

\begin{tabular}{|c|c|c|c|c|}
\hline Number of model version & 1 & 2 & 3 & 4 \\
\hline \multicolumn{5}{|l|}{ geomagnetic latitude of FACs (deg) } \\
\hline zone 1 & 75 & 75 & 75 & 75 \\
\hline zone 2 & 70 & 65 & 65 & 65 \\
\hline \multicolumn{5}{|l|}{ MLT maxima of FAC intensity (h) } \\
\hline zone 1 & $08 \& 20$ & $03 \& 21$ & $01 \& 21$ & $04 \& 20$ \\
\hline zone 2 & $08 \& 20$ & $03 \& 21$ & $04 \& 19$ & $02 \& 19$ \\
\hline \multicolumn{5}{|l|}{ MLT extent of inflowing FACs (h) } \\
\hline zone 1 & $00-12$ & $00-06$ & $00-02$ & $23-06$ \\
\hline zone 2 & $12-24$ & $18-24$ & $18-24$ & $16-24$ \\
\hline \multicolumn{5}{|l|}{ MLT extent of outflowing FACs (h) } \\
\hline zone 1 & $12-24$ & $18-24$ & $18-24$ & $17-23$ \\
\hline zone 2 & $00-12$ & $00-06$ & $00-06$ & $00-04$ \\
\hline \multicolumn{5}{|l|}{ Maxima of FAC intensity $\left(10^{-7} \mathrm{Am}^{-2}\right)$} \\
\hline zone 1 & 1 & 2.5 & 2.5 & 2.5 \\
\hline zone 2 & 0.5 & 1.25 & 1.25 & 1.25 \\
\hline Cross polar cap potential drop $(\mathrm{kV})$ & 10 & 15 & 18 & 22 \\
\hline
\end{tabular}

and NmF2 above EISCAT. The mean value of $F_{10.7}$ was about 200 for this period and the electric field observations showed values of about $10 \mathrm{mV} / \mathrm{m}$ in the evening sector and even smaller values in the noon sector. The diurnal variation of the electric field and $\mathrm{NmF} 2$ for this particular day are close to the average values for the period under study.

Four model runs were performed. The first corresponds to FACs with maximum intensities in the meridional plane of $08-20 \mathrm{MLT}$ and with a cross polar cap potential drop of about $10 \mathrm{kV}$. This ionospheric convection stands for the so-called 'classical' two-cell ionospheric convection pattern driven by the two concentric FAC rings (at $75^{\circ}$ and $70^{\circ}$ geomagnetic latitude) which are approximately the same as those predicted by a simplified consideration of the results of Iijima and Potemra (1976).

The second run assumes FAC maximum intensities in the night-time sector only. In this case the cross polar cap potential drop reached a value of about $15 \mathrm{kV}$. In this case we attempted to obtain an ionospheric convection pattern which is close to the statistical model of the electric field above EISCAT by Senior et al. (1990) and FAC spatial distributions which are similar to those of Fontaine and Peymirat (1996).

In the third and fourth model run the maximum FAC intensities are the same as in the previous case except for small temporal shifts of the FAC zones. The cross polar cap potential drops for these model runs reached values of about 18 and $22 \mathrm{kV}$, respectively. In these cases we investigated the sensitivity of the diurnal variation of $\mathrm{NmF} 2$ and neutral-wind velocities on the spatial variation of the FAC distributions in detail.

The model results are presented in the Figs 2-4 for the first, Figs 5-6 for the second and in Figs 7-9 for the third and fourth run. All components of the vector quantities are presented in the geomagnetic spherical coordinate system. Every model run was analysed with respect to the different origin of the electric field contributions. In the figures the contributions from electric fields of magnetospheric origin only are indicat- ed by open symbols while full symbols illustrate the effect of the full electric fields originating from magnetospheric and dynamo electric fields.

The FAC distributions for the first case as a function of magnetic local time are presented in Fig. 2 (top panel) while the diurnal variations of the electric field components (zonal and meridional) and of $\mathrm{NmF} 2$ for these cases are displayed in the other three panels of Fig. 2 as a function of local time. The amplitudes of the electric field components for the first case are small during the whole day and they reach maximum values of about 1 $\mathrm{mV} / \mathrm{m}$ (corresponding to $20 \mathrm{~m} / \mathrm{s}$ drift velocities). The contribution from magnetospheric electric fields only (open symbols) to the eastward component (Fig. 2, second panel) is smaller than $0.5 \mathrm{mV} / \mathrm{m}$. The inclusion of dynamo electric field effects (full symbols) increases the amplitudes in the morning sector around $07 \mathrm{LT}$ and in the evening sector around 19 LT.

The northward component of the electric field of magnetospheric origin as shown in Fig. 2 (third panel) by downward pointing open triangels reaches values of about $1 \mathrm{mV} / \mathrm{m}$ during the post-noon hours. Here, the dynamo electric field contribution (full triangles) reduces the magnetospheric electric field considerably during the whole day. The total northward electric field component is negative almost throughout the whole day and reaches values of $-1 \mathrm{mV} / \mathrm{m}$ during the morning and evening hours. This component is covered by the range of the experimental errors but the model northward electric field component is smaller than EISCAT data and has no specific time variation in the evening sector. The diurnal variations of NmF2 (Fig. 2, bottom) show a rather good coincidence with the observational EISCAT data (asterisks) for 10-22 LT.

The meridional and zonal components of the neutralwind velocity at $140 \mathrm{~km}$ altitude for the first model run are presented in Fig. 3. One can see that the effect of the dynamo electric field is very small here for the meridional wind component (upper panel) but it is more clearly marked in the zonal wind component (bottom panel). The effect of the dynamo electric field consists in 
Model calculations: field-aligned currents, electric fields and $\mathrm{NmF2}$
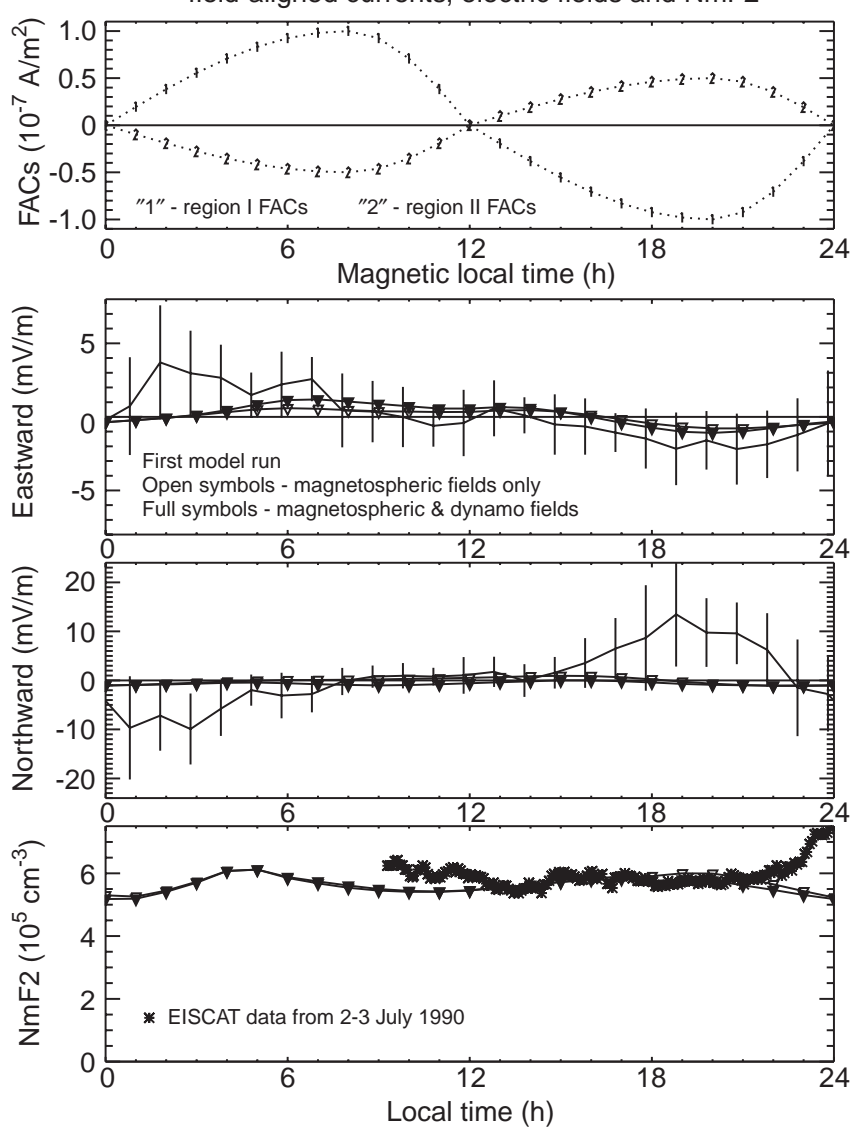

Fig. 2. Variations of the assumed FAC distributions versus MLT (upper panel) and of the eastward and northward electric field components (middle panels), as well as the maximum electron density $\mathrm{NmF} 2$ in the F2 layer (bottom) versus local time; illustrating the first model run in two versions representing magnetospheric electric field action only (open symbols) and magnetospheric and dynamo electric fields together (full symbols)

an increase of the zonal component with an amplitude around $10 \mathrm{~m} / \mathrm{s}$ near $12 \mathrm{LT}$. It can be seen that the neutral-wind velocity at $140 \mathrm{~km}$ altitude has a predominant diurnal component only in this case.

Figure 4 illustrates the electric field potential distributions at the northern hemisphere and the corresponding $\mathrm{NmF} 2$ distributions for the first model run. As one can see in the upper panels, the potential pattern has a well-established two-cell shape and a symmetric potential drop from dawn to dusk. The NmF2 spatial distribution has a slightly marked trough near midnight, i.e. the $N_{e}$ distribution is almost homogeneous throughout the whole polar region.

The two bottom panels of Fig. 4 illustrate the corresponding potential distributions for the same model run but in its version with magnetospheric electric fields only. It is obvious that the electric potential distribution is different from the former version while the NmF2 distribution remains nearly unchanged. The minimum amplitude of the electrical potential is much larger and the ionospheric convection is restricted to high latitudes only.

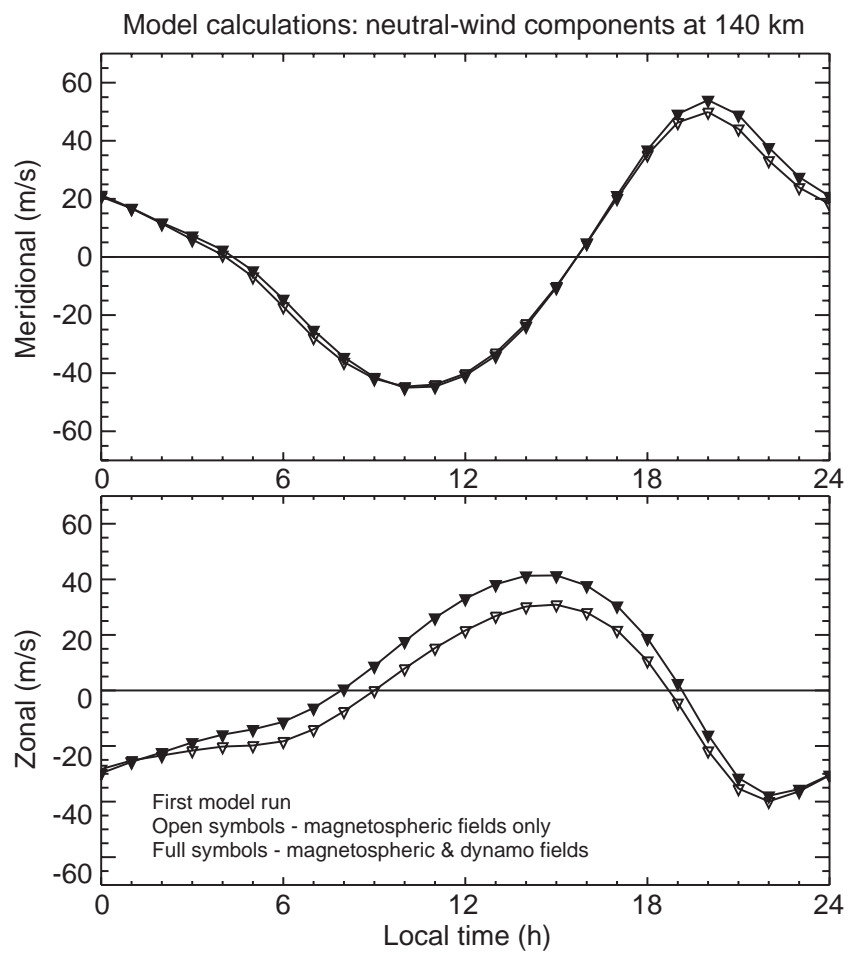

Fig. 3. Local time variations of the neutral wind velocities at E-region height $(140 \mathrm{~km})$ with the meridional (upper panel) and zonal component (bottom) for the same model run as in Fig. 2

Figure 5 shows the FAC variation as a function of MLT (top panel) as well as the electric field components and the variation of $\mathrm{NmF} 2$ versus local time for the second model run. This case constitutes the basic model run for the following trials. As one can see in Fig. 5, the eastward component of the electric field above EISCAT which comes from the contribution of magnetospheric electric fields only (open symbols) is small during 04-15 hours LT and shows a small increase in the evening sector reaching maximum values of about $-2.5 \mathrm{mV} / \mathrm{m}$ at 19 LT. The inclusion of the dynamo electric field (full symbols) changes slightly the behaviour of the electric field in the morning sector. The northward electric field component as displayed in Fig. 5 (third panel) is small between $06 \mathrm{LT}$ and $15 \mathrm{LT}$ and increases during the afternoon and evening hours reaching maximum values at $18-19 \mathrm{LT}(8 \mathrm{mV} / \mathrm{m})$ and $01 \mathrm{LT}(-5 \mathrm{mV} / \mathrm{m})$. The dynamo electric field action diminishes the amplitude of the magnetospheric electric field between 06 LT and 14 LT by about $1 \mathrm{mV} / \mathrm{m}$.

The corresponding calculation results of the neutralwind component at $140 \mathrm{~km}$ altitude are presented in Fig. 6 . The effect of the dynamo electric field is more obvious for the zonal wind component and amounts to $10 \mathrm{~m} / \mathrm{s}$ in the daytime sector. It should be noted here that the zonal wind component at $140 \mathrm{~km}$ altitude has a clearly defined semidiurnal variation.

The comparision of the model electric field components for the total electric field obtained in the second model run and the average EISCAT data observations for the electric field are presented in Fig. 5 (2nd and 3rd 
Potential (kV) $\quad$ First model run
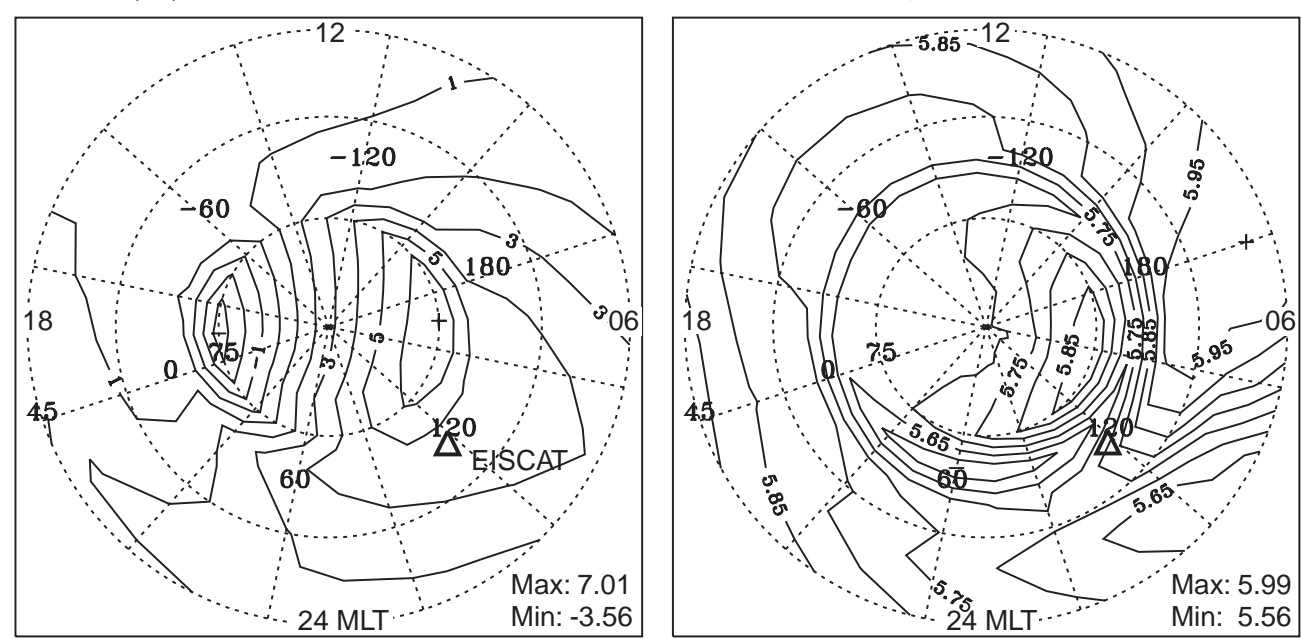

Magnetospheric fields only
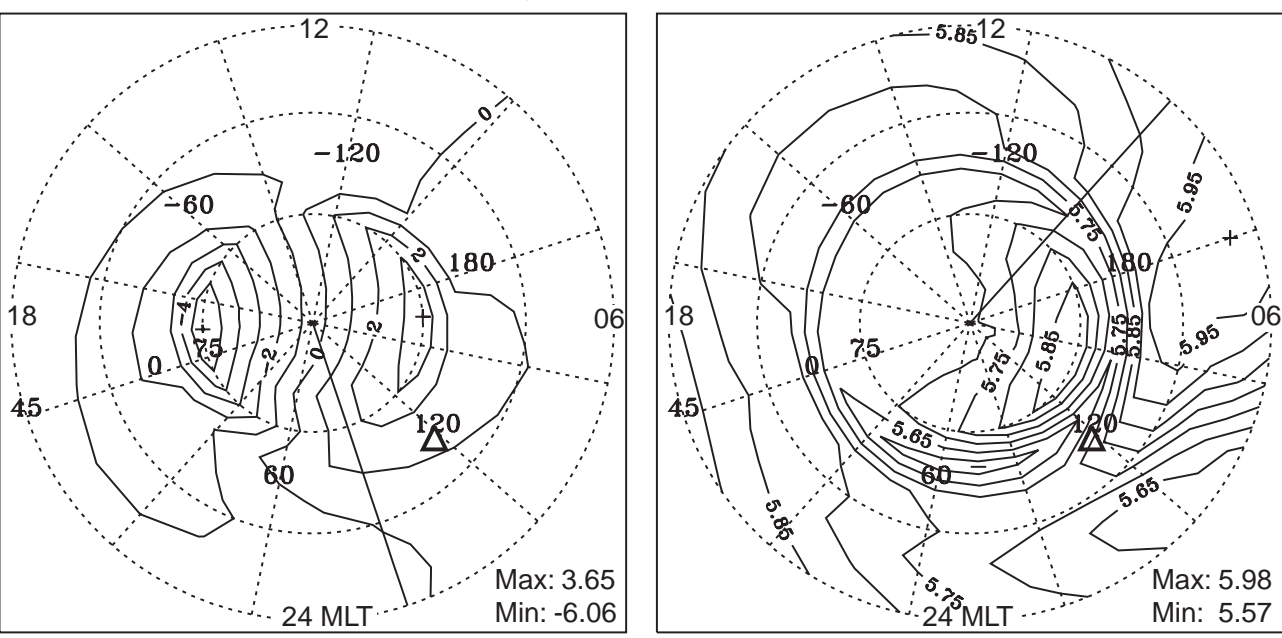

Fig. 4. Potential distributions (left panels) and isolines of $\log (\mathrm{NmF} 2)$ per $\mathrm{cm}^{-3}$ (right) for the first model run. The upper panels illustrate the version with magnetospheric and dynamo electric fields while the bottom panels illustrate the version with the action of magnetospheric electric fields only. The polar plots show the northern hemisphere distributions at $00 \mathrm{UT}$ with magnetic local time and magnetic latitude (down to $45^{\circ}$ ) as coordinates. The EISCAT station location is indicated by a thick triangle panels) also. It can be seen that the northward electric field component is in agreement in amplitude and phase with the experimental data. The eastward component of the electric field show also a good agreement with EISCAT data on average (about zero).

Modifications of the second, basic model run have been done in the third and fourth runs varying the spatial FAC distributions but not their amplitudes. Figure 7 shows the FAC distributions (upper panel), the corresponding electric field components (middle panels) and its effects on the diurnal variation of NmF2 above EISCAT. The basic version (second model run) is marked by squares while the two others are marked by circles and diamonds for the third and fourth case, respectively.

As for the third model run one can see that the eastward electric field component has an amplitude of about $\pm 3 \mathrm{mV} / \mathrm{m}$ and the northward component maximizes at $18 \mathrm{LT}$ and has zero values at 06 and $24 \mathrm{LT}$. The NmF2 diurnal variation shows slightly smaller values in the morning sector and larger values after sunset.

In the fourth model run, finally, the plot shows an opposite variation with similar amplitudes of the eastward electric field component in comparison with the former run almost during the whole day. The northward electric field component has a maximum at $18 \mathrm{LT}$ as in the third run but a zero value near $21 \mathrm{LT}$. The diurnal variation of $\mathrm{NmF} 2$ (bottom panel) shows a strong depletion of $N_{e}$ in the evening sector. The comparison of these three different versions of the third model run demonstrates the effects of a mutual shift in time of the location of the FAC regions on the diurnal variation of NmF2 above EISCAT.

This is also illustrated in the following Fig. 8 with respect to the neutral-wind components at 140 and 279 $\mathrm{km}$ altitude. Due to the different convection pattern, the amplitudes of the neutral-wind components at $140 \mathrm{~km}$ altitude can vary as much as $50 \mathrm{~m} / \mathrm{s}$ for the different versions (2nd-4th model run). Besides the general increase in the electric field potential drop from about $18 \mathrm{kV}$ (3rd run) to $22 \mathrm{kV}$ (4th), the results show an increase in the meridional wind amplitude and a decrease in the mean zonal wind amplitude at $140 \mathrm{~km}$ altitude. This effect can also be seen at $279 \mathrm{~km}$ altitude.

Figure 9, finally, shows the potential patterns and the corresponding spatial NmF2 distributions at the northen hemisphere for all three versions (model run 2 to 4 ). The basic case (second model run) is displayed at the top 
Model calculations:

field-aligned currents, electric fields and $\mathrm{NmF2}$
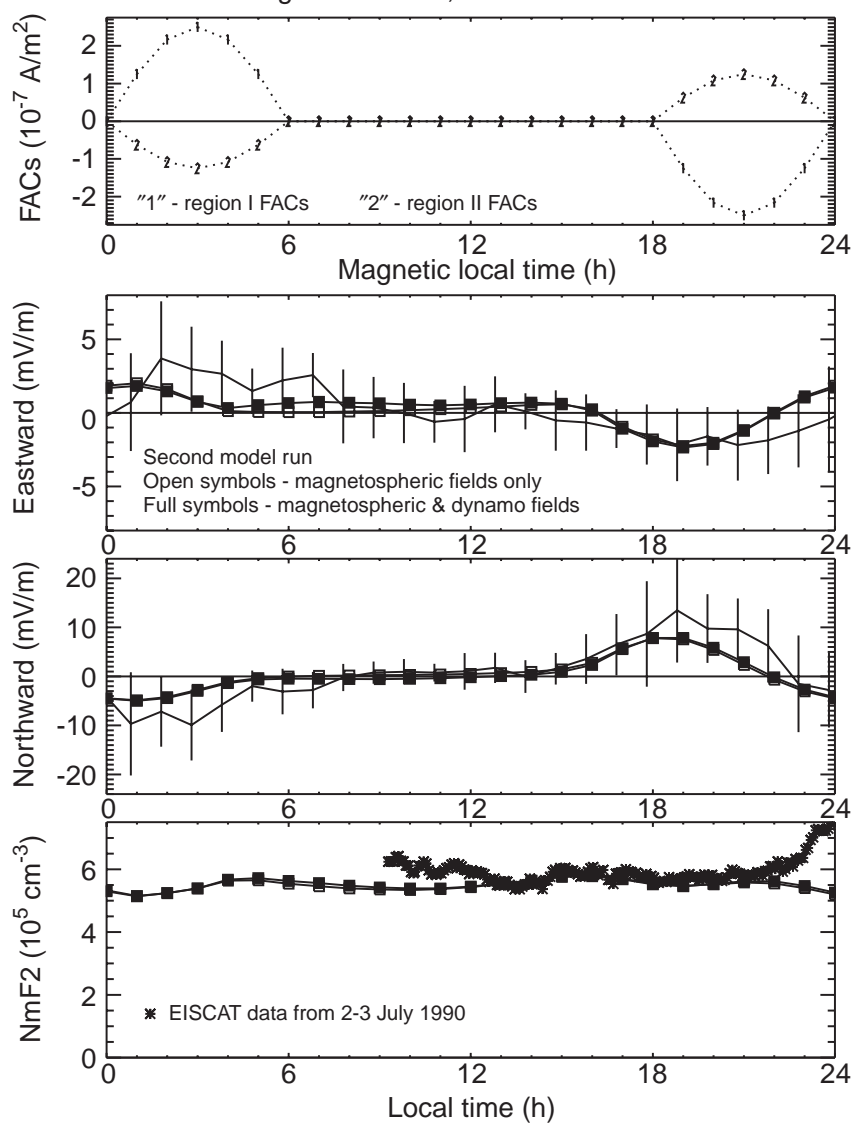

Fig. 5. The same representation as in Fig. 2, but for the second (or 'basic') model run with FAC distribution (upper panel), electric field components (middle) and NmF2 (bottom)

panel. The cross polar cap potential drop for this version is about $15 \mathrm{kV}$. The two-cell convection pattern is located mainly in the night-time sector and the spatial distribution of $\log (\mathrm{NmF} 2)$ (per $\mathrm{cm}^{-3}$ ) varies slightly from about 5.50 near midnight to 5.95 at 06 MLT. In version three the cross potential drop increased to $18 \mathrm{kV}$ and the minimum $\mathrm{NmF} 2$ is now $\log (\mathrm{NmF} 2) \sim 5.58$. Panel (c) in Fig. 9 shows a potential pattern with an increasing cross polar cap potential drop of up to $22 \mathrm{kV}$ for the same intensities of the FACs but with shifted spatial location. In this case a deep trough of the NmF2 distribution can be observed near midnight with $\log (\mathrm{NmF} 2) \sim 4.85$. The EISCAT station is located at the morning side of the $N_{e}$ depletion.

\section{Discussion}

In the previous section we presented model results obtained with four different sets of input parameters causing different magnetospheric convection patterns, calculated with and without the inclusion of dynamo electric fields. From experimental observations of the drift velocities above EISCAT for geomagnetically quiet conditions during summer solstice and moderate to high

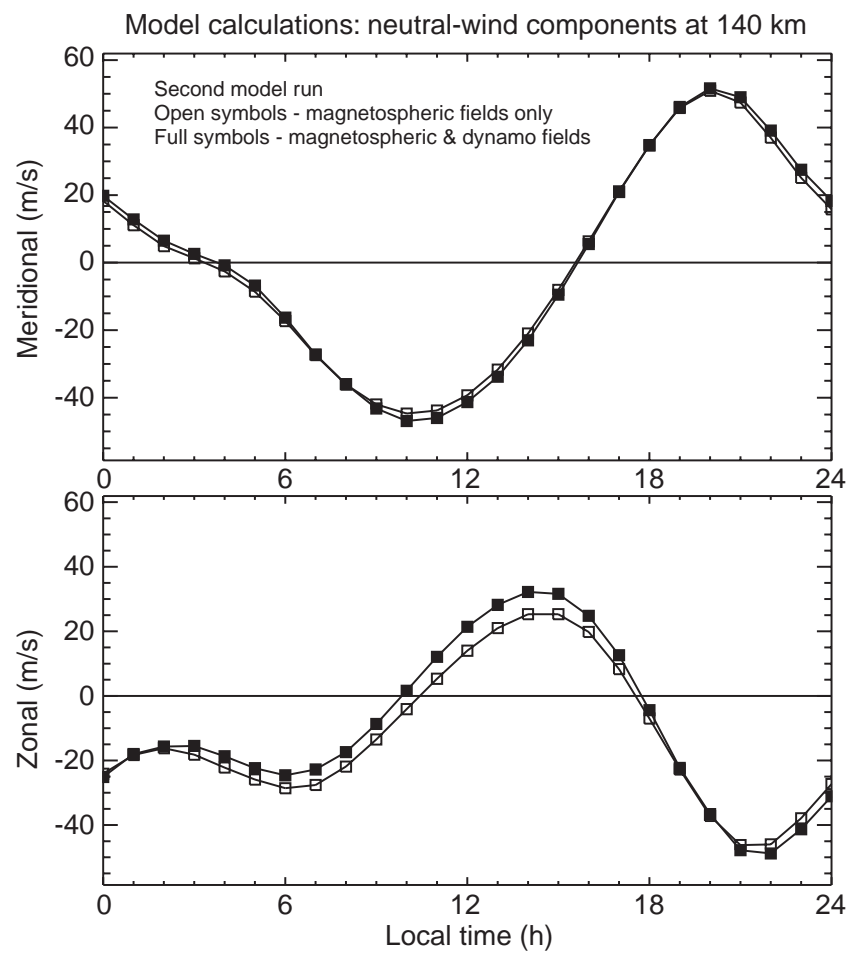

Fig. 6. The same representation as in Fig. 3, but for the second ('basic') model run with the meridional and zonal neutral wind components for $140 \mathrm{~km}$ versus local time

solar activity conditions we concluded that the electric field components near the EISCAT station are rather small during daytime hours but as far as the zonal drift component is concerned the velocity can reach a few hundreds $\mathrm{m} / \mathrm{s}$ during evening and night-time hours only. This conclusion is confirmed by the study of Brekke et al. (1994). They presented averaged electric field components for seven geomagnetically quiet days in summer together with the statistical convection electric field model values of Senior et al. (1990).

Therefore we can suppose that the dynamo electric fields can have a marked effect on thermosphere/ ionosphere parameters above EISCAT in the daytime sector. In order to study this effect we have executed the two aforementioned different model runs: the first for a very small electric field with a symmetrical distribution of FACs (classic convection) and the second, a typical case for geomagnetically quiet times with a potential drop of about $15-20 \mathrm{kV}$.

In the first case, the model zonal electric field component is small (about zero) and within the error bars of the experimental data. The northern component of the electric field (zonal drift velocity) is much smaller than the averaged EISCAT data in the evening sector (Fig. 2). There one can notice also that the dynamo electric field makes a contribution to the total electric field above EISCAT in the case of the smallest cross potential drop in the polar region. The effect of the dynamo electric field on the meridional neutral wind component at $140 \mathrm{~km}$ altitude is small in this case, but there is an appreciable contribution to the zonal neutral 
Model calculations: field-aligned currents, electric fields and $\mathrm{NmF2}$
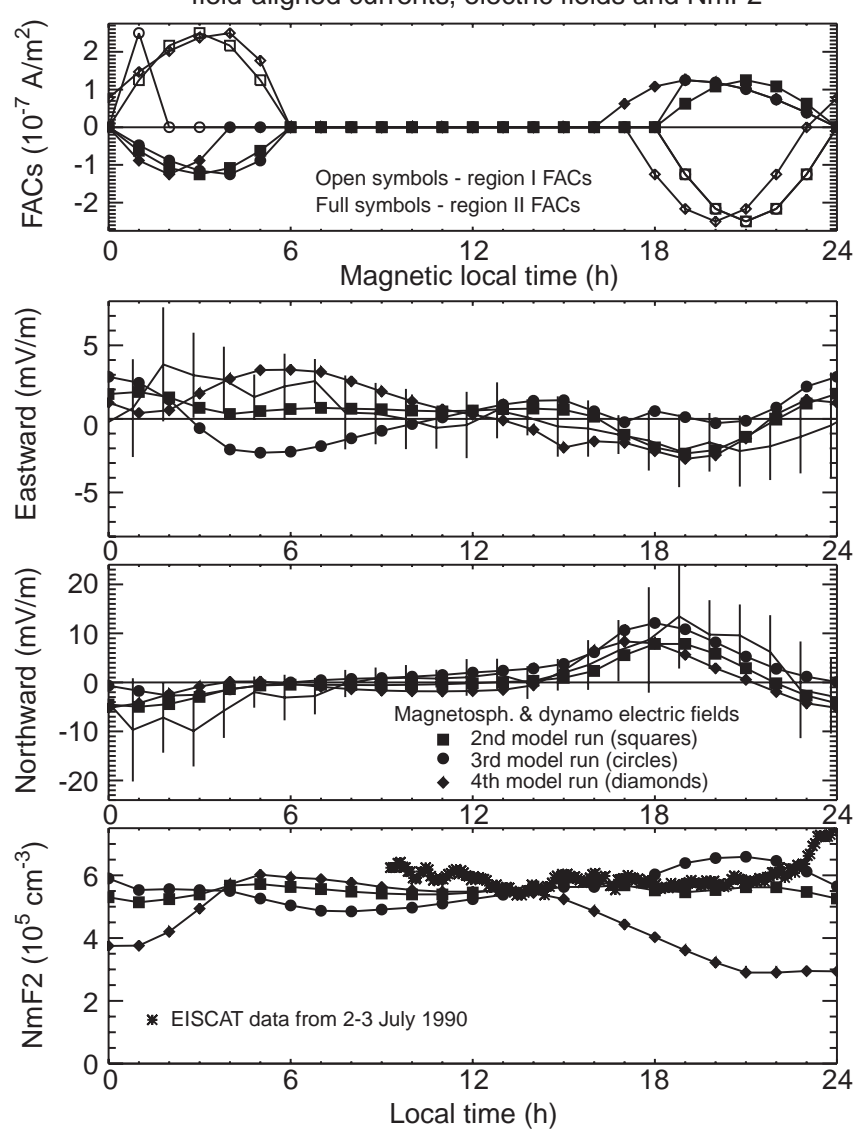

Fig. 7. The same representation as in Figs. 2 and 5, but for the 2nd to 4th model runs with FAC distributions (upper panel), electric field components (middle) and $\mathrm{NmF} 2$ variations (bottom)

wind component (Fig. 3). The eastward wind component increases by about $10 \mathrm{~m} / \mathrm{s}$ during almost the whole day. It can be noticed that the eastward component has a predominant diurnal variation in this case.

Small electric fields cause small ion drift velocities in the ionospheric $\mathrm{F}$ region and the agreement between modelled and observed NmF2 is reasonably good for this case (Fig. 2, bottom panel) although the meridional electric field component of the model results differs in amplitude from the data. As we can see in this case the symmetric or 'classic' ionospheric convection does not form the diurnal variation of the northward electric field component as it was obtained by Senior et al. (1990) during geomagnetically quiet conditions, but the cross polar cap potential drop $(10 \mathrm{kV})$ is close to the statistical model result $(12.4 \mathrm{kV})$. The agreement between the calculated and observed $\mathrm{NmF} 2$ values may be explained by the small effects brought about by the ionospheric convection under these conditions and by the dominance of solar radiation in summer when the whole polar region is sunlit.

In order to obtain an agreement between the statistical model of Senior et al. (1990) and the model results we have chosen a second convection pattern controlled by a specific FAC distribution shown in Fig.5 (upper
Model calculations: neutral-wind components at 140 and $279 \mathrm{~km}$
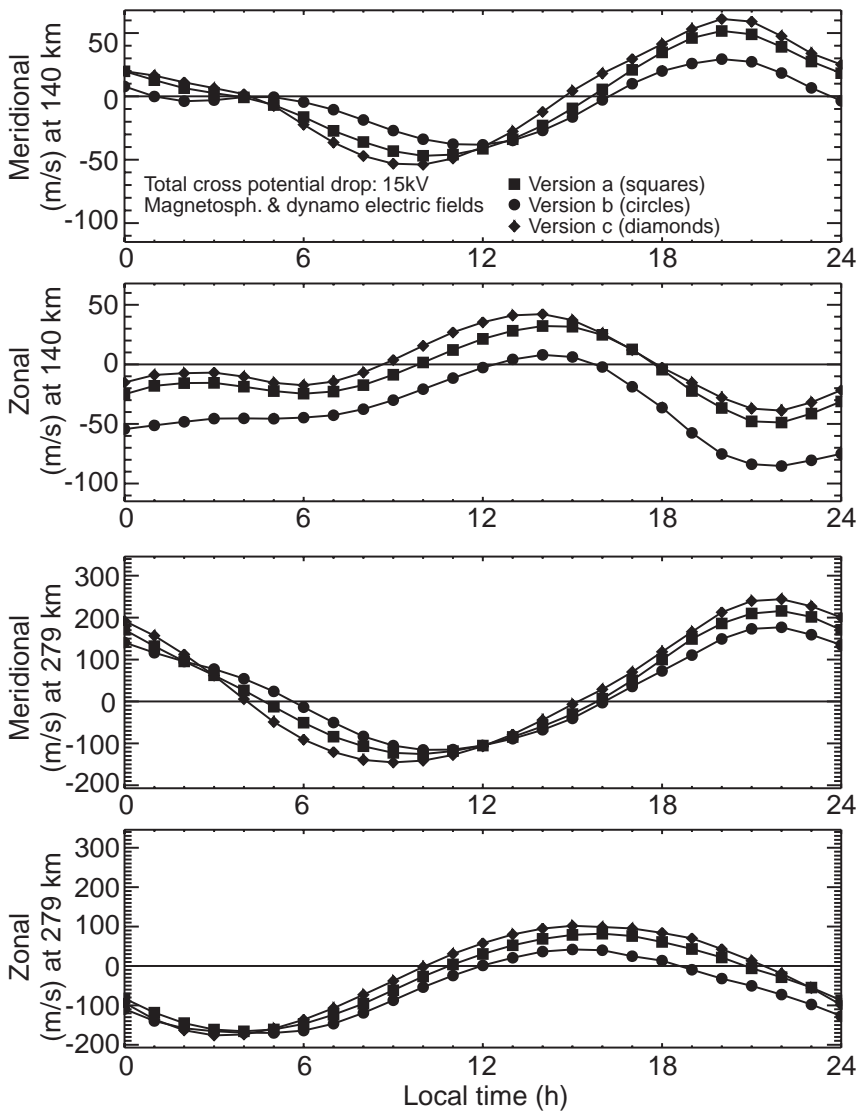

Fig. 8. As in Figs. 3 and 6, but for three different model runs (2nd to 4 th) and demonstrating the meridional and zonal neutral wind components for $140 \mathrm{~km}$ (upper two panels) and $279 \mathrm{~km}$ height (bottom) versus local time

panel). The maximum FAC intensities are reached at 03 and 21 MLT. In this second model run we moved the region-II FACs towards lower latitudes at $65^{\circ}$. A similar FAC distribution was obtained by Caudal (1987) and Fontaine and Peymirat (1996). One should keep in mind that these latest results may involve a considerable error in the estimation of the FAC location for very quiet geomagnetic activity conditions because the current densities are very weak under these circumstances and the authors of these papers have neglected neutral-wind effects as caused, e.g. by the dynamo electric field. Nevertheless, our FAC distribution (see Fig. 5, upper panel) is in qualitative agreement with the aforementioned study of Fontaine and Peymirat (1996). The calculated convection pattern resulting from this FAC distribution is similar to the model results obtained by Kamide and Matsushita (1979) and Senior et al. (1990) for very quiet geomagnetic conditions while the model cross polar cap potential drop of about $15 \mathrm{kV}$ is a little bit larger than the $12.4 \mathrm{kV}$ as presented by Senior et al. (1990). As has been shown in Fig. 5, the qualitative agreement between model electric field components and experimental data is reasonably good in this case. But one should notice that the eastward electric field component may be compared with the experimental 

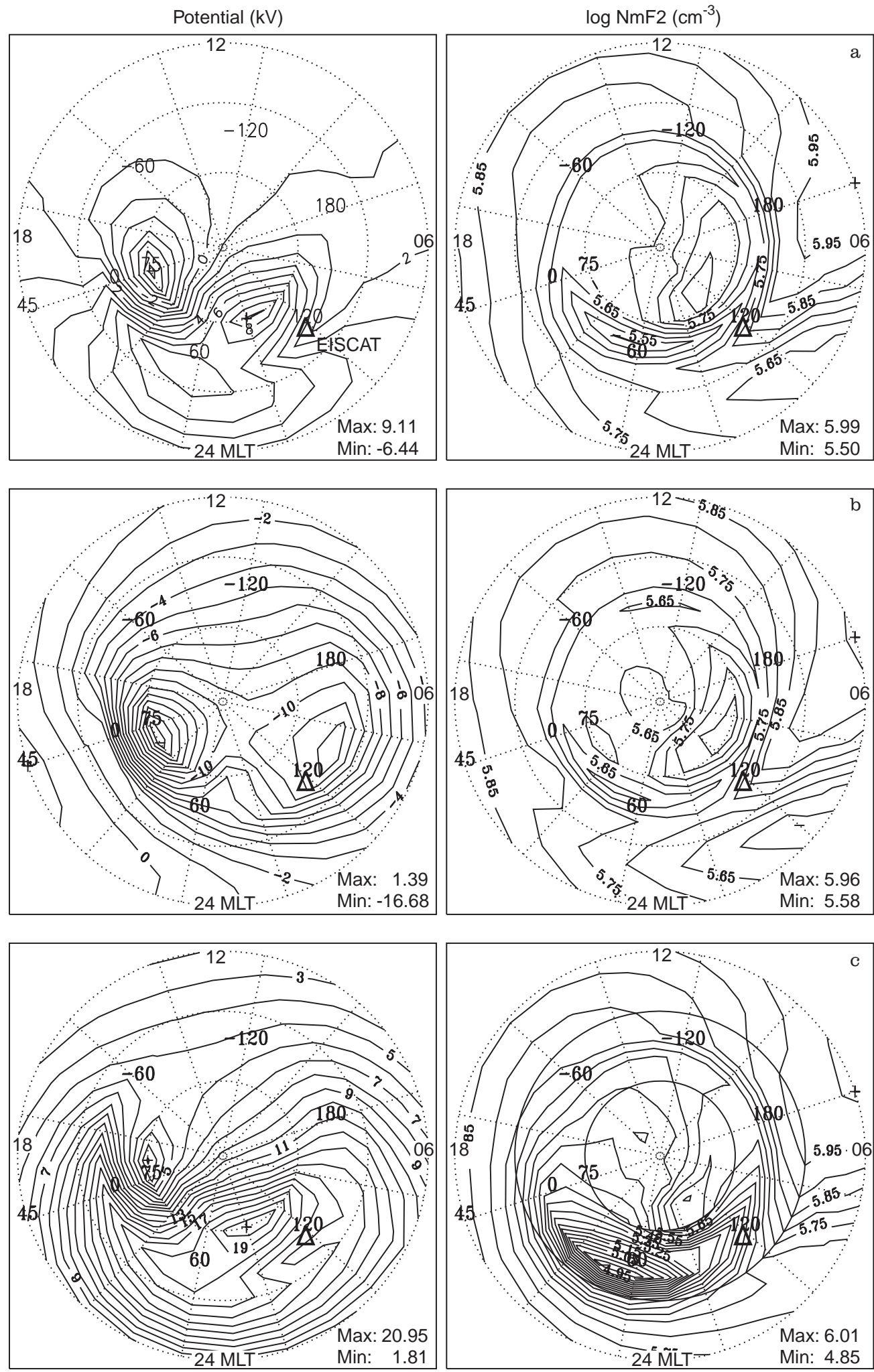

Fig. 9. The same representation as in Fig. 4, but for the three model runs (2nd, 3 rd and 4 th according Table 2) shown from top to bottom as case a-c. The left panels show electric potential distributions and the right panels the isolines of $\log (\mathrm{NmF} 2)$ per $\mathrm{cm}^{-3}$. The polar plots are for the northern hemisphere at $00 \mathrm{UT}$ with magnetic local time and magnetic latitude (down to $45^{\circ}$ ) as coordinates. The EISCAT station location is indicated by a thick triangle

data under consideration of the r.m.s. bars given. Thus we may conclude that the meridional electric field component prevails over the zonal one above EISCAT for all cases under quiet geomagnetic conditions in the evening sector (see Fig. 1 and Brekke et al., 1994; Senior et al., 1990). One should notice that the agreement between the model northward electric field component and the average experimental data may be obtained only with an assymetric FAC distribution. The amplitude of the northward model electric field is smaller than the average data, but it is in the range of the STD of the measured values. The phase parameter of the meridional electric field component is in good agreement with the experimental data as well. 
The model results show that in the second case the zonal wind component (Fig. 6, bottom) has a marked semidiurnal variation at $140 \mathrm{~km}$ altitude in contrary to the diurnal variation of the first case with smaller electric fields (Fig. 3). We used constant values at the lower boundary for the neutral atmosphere parameters for the solution of the corresponding equations, i.e. tides propagating from the middle atmosphere were not taken into account. Thus, the semidiurnal variation of the zonal wind component at $140 \mathrm{~km}$ altitude is caused by magnetospheric convection via the interaction between ion and neutral species and their different velocities. The combination of the internal (or inherent) diurnal thermosphere variation with the wind variation generated by the magnetospheric convection give rise to the semidiurnal wind mode at $140 \mathrm{~km}$ altitude. This wind mode is not connected with natural tidal modes propagating from the mesosphere. The effect of tidal mode excitations in the thermosphere by electric fields was mentioned by Brekke et al. (1994). But we should note here that in our model calculations only semidiurnal variations of the E-region zonal wind component were found and this variations are caused by the semidiurnal mode of the northward electric field component above EISCAT.

It is known from tidal theory and observations (see, e.g., Forbes and Vial, 1989) that the amplitudes of the propagating solar semidiurnal tides are small at the high-latitude mesosphere $(80 \mathrm{~km})$, but they are clearly observed at E-region heights above EISCAT. Some results of such analyses were presented in the papers of Williams and Virdi (1989), Brekke et al. (1994), Kofman et al. (1996) and others. As has been shown in these studies the amplitude of the semidiurnal tide is maximal at about $110 \mathrm{~km}$ altitude with values of about $50 \mathrm{~m} / \mathrm{s}$. Our model wind amplitudes are somewhat smaller than the experimental data. Thus we may conclude that the mesospheric tides play a significant role in the wind structure of the lower thermosphere. At the same time the semidiurnal mode generated by the ionospheric convection in the second model run is quantatively in agreement with experimental data and produces about $30 \%$ of the measured semidiurnal tide amplitude.

As we have shown with the model results for the second case the amplitude and phase of the electric field components are in qualitative agreement with experimental data for both components and the cross polar cap potential drop with a value of about $15 \mathrm{kV}$ is typical for quiet geomagnetic conditions. At the same time the diurnal variation of $\mathrm{NmF} 2$ in this case (Fig. 5, bottom) shows an agreement with EISCAT data as well.

In order to investigate the sensitivity of the time variation of the NmF2 in the evening/night-time sector to the phase parameter of the northward electric field (eastward drift) component we introduced an asymmetric MLT shift for the maximum FAC intensities of regions I and II for the third and fourth model runs (see Fig. 7). It is obvious that the shift of the northward electric field component (eastward drift) toward earlier evening hours is accompanied by a depletion of $N_{e}$ in the F2 region above EISCAT (Fig. 7, 4th version) and the shift toward midnight causes a slight increase of $\mathrm{NmF} 2$ during the pre-midnight hours (3rd model run). These effects are connected with the combined action of the eastward and the northward plasma drift as has been discussed by Klimenko and Namgaladze (1977). It can also clearly be seen in Fig. 9 that larger $\mathrm{NmF} 2$ values are connected with drift trajectories which are passing near the EISCAT station coming from a region with rather high $N_{e}$ densities ( 3 rd run) while for the opposite case (4th run) the drift trajectories come from the ionization trough located near the midnight sector. A more detailed analysis of the diurnal electric field variations above EISCAT has shown that the depletion of NmF2 is connected with the change of the eastward electric field component. The electron density increases when the eastward component is near or equal zero, and it decreases when this component is negative while the northward electric field component is positive in both cases (see Fig. 7). Thus we may conclude that the small eastward electric field component plays a significant role in the spatial/time distribution of the $\mathrm{NmF} 2$ above EISCAT.

It can be concluded from the foregoing discussion that the results for the second model run with a cross potential drop of $15 \mathrm{kV}$ and asymmetrical maximum FAC intensities in the meridional planes of 03 and 21 MLT confirm the statistical models of Senior et al. (1990) and Fontaine and Peymirat (1996) obtained by use of experimental EISCAT data.

Finally, we examined the effect of the various locations of the FAC regions on the diurnal variation of the neutral-wind components at 140 and $279 \mathrm{~km}$ altitude (Fig. 8). These effects differ considerably from the effects of the dynamo electric field on the neutralwind velocities. The increase in the total potential drop from $18 \mathrm{kV}$ (3rd case) to $22 \mathrm{kV}$ (4th) causes an increase in the meridional wind amplitude at all altitudes without any phase variations. At the same time there is a decrease in the mean zonal wind, which is especially obvious at $140 \mathrm{~km}$ altitude. These wind variations are caused by the influence of the ionospheric convection via ion drift interactions with neutral-wind flows.

\section{Summary and conclusions}

In this paper, the thermosphere and ionosphere effects of the electric field of both magnetospheric and dynamo origin have been modelled using the global numerical model of the coupled thermosphere ionosphere and protonosphere (GSM TIP). The calculations were executed for summer solstice, high solar activity and quiet geomagnetic conditions and compared with corresponding averaged EISCAT electric field and $\mathrm{NmF} 2$ measurements for summer and high to medium solar activity conditions.

Four model situations were studied. The first one with a very small cross polar potential drop of about $10 \mathrm{kV}$ and a symmetric FAC distribution corresponds to the 'classic' ionospheric convection. The second 'most realistic case' has a cross potential drop of $15 \mathrm{kV}$ and an 
asymmetric FAC distribution. For these cases the model runs were executed twice - with and without dynamo electric fields - to highlight their effects on the thermosphere/ionosphere parameters. In the third and fourth model run we introduced an asymmetric time-shift for the maximum FAC intensities of regions I and II. This led to some increase in the total potential drop from $18 \mathrm{kV}$ (3rd case) to $22 \mathrm{kV}$ (4th) in these model runs.

We have shown that in the first case the electric field components are about $\pm 1 \mathrm{mV} / \mathrm{m}$ during the whole day and with consideration of the uncertainties of the experimental data they generally agree with the averaged EISCAT observations for this period under study except for the evening to night-time hours. Neutral wind components at $140 \mathrm{~km}$ altitude have a diurnal variation only, and the effect of the dynamo electric field consists in increasing the zonal wind amplitude during daytime by values of about $10 \mathrm{~m} / \mathrm{s}$. Comparisions of the modelled and observed NmF2 above EISCAT have shown a qualitative agreement already for the first model run.

In the second case we obtained a qualitative agreement between model and the averaged experimental electric field data, and a reasonable agreement with the diurnal NmF2 variation for the EISCAT measurements of 2-3 July 1990.

We also found that the meridional wind component at $140 \mathrm{~km}$ altitude has a diurnal variation only (for the second case), but the zonal wind component modified by the action of magnetospheric convection has a semidiurnal variation. This oscillation is not connected with the usual tidal propagation from the mesosphere. It results from a combination of the global thermospheric circulation with the wind variation generated by the polar cap drifts via the thermosphere/ionosphere interaction through ion-neutral collisions.

The effect of dynamo electric fields is clearly observed in the zonal wind component at $140 \mathrm{~km}$ altitude and consists in an enhanced wind amplitude by about $10 \mathrm{~m} / \mathrm{s}$ during daytime hours.

We have studied the effects of various FAC locations on the thermosphere/ionosphere parameters. It was shown that averaged statistical models of the convection pattern can be confirmed with FAC zones at $75^{\circ}$ (region I) and $65^{\circ}$ (region II) geomagnetic latitudes. The maximum FAC intensities have been assumed in the meridional planes of 03-21 MLT for both regions and are equal to $2.5 \times 10^{-7} \mathrm{~A} \mathrm{~m}^{-2}$ (region I) and $1.25 \times 10^{-7} \mathrm{~A} \mathrm{~m}^{-2}$ (region II). In this case the cross polar cap potential drop is about $15 \mathrm{kV}$ with a two-cell convection pattern situated mainly in the night-time sector which is also confirmed by various statistical potential models constructed with the use of EISCAT data for quiet geomagnetic conditions.

A reasonable agreement with the EISCAT drift data and with the experimental diurnal variation of $\mathrm{NmF} 2$ was obtained in this model run as well. Our investigations have shown that the electron concentration above EISCAT is very sensitive with respect to the magnetospheric convection pattern in the pre-midnight timespan. It may cause an increase or decrease in $\mathrm{NmF} 2$ near
20-22 LT according to the FAC maximum intensity locations. The neutral-wind components are affected by the ionospheric convection as well. The deviation of the wind amplitude reached about $50 \mathrm{~m} / \mathrm{s}$ for both wind components at 140 and $279 \mathrm{~km}$ for the different versions of the FAC locations.

Acknowledgements. We are grateful to K. Schlegel (MPAE, Lindau-Katlenburg) for providing EISCAT data. This work was supported by RFFI grant 96-05-64182.

Topical Editor D. Alcaydé thanks A. D. Richmond and another referee for their help in evaluating this paper.

\section{References}

Blelly, P. L., J. Lilensten, A. Robineau, J. Fontanari, and D. Alcaydé, Calibration of a numerical ionospheric model with EISCAT observations, Ann. Geophysicae, 14, 1375-1390, 1996a.

Blelly, P. L., A. Robineau, and D. Alcaydé, Numerical modelling of sporadic ion outflow events above EISCAT, J. Atmos. Terr. Phys., 58, 273-285, 1996b.

Brekke, A. S., S. Nozava, and T. Sparr, Studies of the E-region neutral wind in the quiet auroral ionosphere, J. Geophys. Res., 99, 8801-8825, 1994.

Caudal, G., Field-aligned currents deduced from EISCAT radar observations and implications concerning the mechanism that produces region 2 currents, J. Geophys. Res., 92, 6000-6012, 1987.

Farmer, A., T. Fuller-Rowell, and S. Quegan, Comparing numerical simulations of the high-latitude ionosphere to an empirical mean model based on EISCAT data, Adv. Space Res., 10(6), 143-148, 1990.

Fontaine, D., and C. Peymirat, Large-scale distributions of ionospheric horizontal and field-aligned currents inferred from EISCAT, Ann. Geophysicae, 14, 1284-1296, 1996.

Fontaine, D., S. Perrau, D. Alcayde, G. Caudal, and B. Higel, Large-scale structures of the convection inferred from coordinated measurements by EISCAT and GEOS-2, J. Atmos. Terr. Phys., 48, 973-986, 1986.

Forbes, J. M., and F. Vial, Monthly simulations of the solar semidiurnal tide in the mesosphere and lower thermosphere, $J$. Atmos. Terr. Phys., 51, 649-661, 1989.

Foster, J. C., Radar-deduced models of the convection electric field in quantitative modelling of magnetosphere-ionosphere processses, pp. 71-76, Kyoto, Japan. American Geophysical Union, 1987.

Hardy, D. A., M. S. Gussenhoven, and E. A. Holeman, A statistical model of auroral electron precipitation, J. Geophys. Res., 90, 4229-4248, 1985.

Iijima, T., and T. A. Potemra, The amplitude distribution of the field-aligned currents at northern high latitudes observed by TRIAD, J. Geophys. Res., 81, 2165-2174, 1976.

Kamide, J. M., and S. Matsushita, Simulation studies of ionospheric fields and currents in relation to field-aligned currents, 1. Quiet period, J. Geophys. Res., 84, 981-987, 1979.

Klimenko, V. V., and A. N. Namgaladze, Ionospherric effects of zonal and meridional electric fields in the evening sector, Geomagn. Aeron., 17, 441-444, 1977.

Kofman, W., C. Lathuillere, and B. Pibaret, Neutral dynamics of high-latitude E-region from EISCAT measurements: a new approach, J. Atmos. Terr. Phys., 58, 121-128, 1996.

Korenkov, Y. N., V. V. Klimenko, M. Förster, V. A. Surotkin, and J. Smilauer, Global modelling study (GSM TIP) of the ionospheric effects of excited $N_{2}$, convection and heat fluxes by comparison with EISCAT and satellite data for 31 July 1990, Ann. Geophysicae, 14, 1362-1374, 1996.

Mount, G. H., and G. J. Rottman, The solar absolute spectral irradiance 1150-3173 А: May 17, 1982, J. Geophys. Res., 88, 5403-5410, 1983. 
Namgaladze, A. A., Y. N. Korenkov, V. V Klimenko, I. V. Karpov, F. S. Bessarab, V. A. Surotkin, T. A. Glushchenko, and N. M. Naumova, Global model of the thermosphere-ionosphere-protonosphere system, Pure Appl. Geophys., 127, 219-254, 1988.

Namgaladze, A. A., Y. N. Korenkov, V. V. Klimenko, I. V. Karpov, F. S. Bessarab, V. A. Surotkin, T. A. Glushchenko, and N. M. Naumova, A global numerical model of the thermosphere, ionosphere, and protonosphere of the earth, Geomagn. Aeron., 30, 512-521, 1990.

Namgaladze, A. A., Y. N. Korenkov, V. V. Klimenko, I. V. Karpov, V. A. Surotkin, and N. M. Naumova, Numerical modelling of the thermosphere-ionosphere-protonosphere system, J. Atmos. Terr. Phys., 53, 1113-1124, 1991.

Namgaladze, A. A., O. V. Martynenko, A. N. Namgaladze, M. A. Volkov, Y. N. Korenkov, V. V. Klimenko, I. V. Karpov, and F. S. Bessarab, Numerical simulation of an ionospheric disturbance over EISCAT using a global ionospheric model, J. Atmos. Terr. Phys., 58, 297-306, 1996.

Nusinov, A. A., Dependence of intensity of lines of shortwave radiation of the Sun on activity level (in Russian), Geomagn. Aeron., 24, 529-536, 1984.

Schoendorf, J., A. D. Aylward, and R. J. Moffett, Modelling highlatitude electron densities with a coupled thermosphere-ionosphere model, Ann. Geophysicae, 14, 1391-1402, 1996.

Senior, C., D. Fontaine, G. Caudal, D. Alcaydé, and J. Fontanari, Convection electric fields and electrostatic potential over 61-72 invariant latitude observed with the European incoherentscatter facility, 2. Statistical results, Ann. Geophysicae, 8, 257272, 1990.

Williams, P. J. S., and T. S. Virdi, EISCAT observations of tidal modes in the lower thermosphere, J. Atmos. Terr. Phys., 51, 569-577, 1989. 